\title{
Retinoic Acid Inhibits Induction of c-Jun Protein by Ultraviolet Radiation that Occurs Subsequent to Activation of Mitogen-activated Protein Kinase Pathways in Human Skin In Vivo
}

\author{
Gary J. Fisher, Harvinder S. Talwar, Jiayuh Lin, Pinpin Lin, Fiona McPhillips, ZengQuan Wang, Xiaoyan Li, Yinsheng Wan, \\ Sewon Kang, and John J. Voorhees \\ Department of Dermatology, University of Michigan Medical School, Ann Arbor, Michigan 48109-0609
}

\begin{abstract}
Human skin is exposed daily to solar ultraviolet (UV) radiation. UV induces the matrix metalloproteinases collagenase, 92-kD gelatinase, and stromelysin, which degrade skin connective tissue and may contribute to premature skin aging (photoaging). Pretreatment of skin with all-trans retinoic acid ( $t$ RA) inhibits UV induction of matrix metalloproteinases. We investigated upstream signal transduction pathways and the mechanism of $t \mathrm{RA}$ inhibition of $\mathrm{UV}$ induction of matrix metalloproteinases in human skin in vivo. Exposure of human skin in vivo to low doses of UV activated EGF receptors, the GTP-binding regulatory protein $\mathrm{p}^{21} \mathrm{Ras}$, and stimulated mitogen-activated protein (MAP) kinases, extracellular signal-regulated kinase (ERK), c-Jun aminoterminal kinase (JNK), and p38. Both JNK and p38 phosphorylated, and thereby activated transcription factors c-Jun and activating transcription factor 2 (ATF-2), which bound to the c-Jun promoter and upregulated c-Jun gene expression. Elevated c-Jun, in association with constitutively expressed c-Fos, formed increased levels of transcription factor activator protein (AP) 1, which is required for transcription of matrix metalloproteinases. Pretreatment of human skin with $t$ RA inhibited UV induction of c-Jun protein and, consequently, AP-1. c-Jun protein inhibition occurred via a posttranscriptional mechanism, since $t \mathrm{RA}$ did not inhibit UV induction of c-Jun mRNA. These data demonstrate, for the first time, activation of MAP kinase pathways in humans in vivo, and reveal a novel posttranscriptional mechanism by which $t$ RA antagonizes UV activation of AP-1 by inhibiting c-Jun protein induction. Inhibition of c-Jun induction likely contributes to the previously reported prevention by $t R A$ of $U V$ induction of AP-1-regulated matrixdegrading metalloproteinases in human skin. (J. Clin. Invest. 1998. 101:1432-1440.) Key words: photoaging • matrix metalloproteinases - activator protein 1 - c-Jun amino-terminal kinase • extracellular signal-regulated kinase
\end{abstract}

All authors contributed equally to this work.

Address correspondence to Gary Fisher, Ph.D., University of Michigan Medical School, Department of Dermatology, 1150 West Medical Center Drive, Medical Science I Building, R 6447, Ann Arbor, MI 48109-0609. Phone: 313-763-1469; FAX: 313-647-0076; E-mail: dianemch@umich.edu

Received for publication 25 October 1997 and accepted in revised form 22 January 1998.

J. Clin. Invest.

(c) The American Society for Clinical Investigation, Inc. 0021-9738/98/03/1432/09 \$2.00

Volume 101, Number 6, March 1998, 1432-1440

http://www.jci.org

\section{Introduction}

The skin and the eyes are the only human organs directly exposed to the environment and therefore vulnerable to ultraviolet (UV) radiation from the sun. UV radiation is subdivided into four wavelength ranges: UVC (<290 nm), UVB (290-320 nm), UVA2 (320-340 nm), and UVA1 (340-400 nm). The damaging biological effects of UV radiation on human skin, such as photoaging and skin cancer, emanate primarily from UVB (1). Wavelengths in the UVA2 range penetrate more deeply into skin than UVB, and also contribute to photoaging (2). UVC and most UVB radiation are absorbed by ozone in the earth's stratosphere.

Accumulating evidence from in vitro studies indicates that UV radiation activates cell surface growth factor and cytokine receptors, and therefore mimics the actions of receptor ligands (3-7). This model helps to explain the diverse array of cellular responses elicited by UV radiation. Activation of growth factor and cytokine receptors stimulates mitogen-activated protein (MAP) $)^{1}$ kinase signal transduction pathways (8-10). Three families of MAP kinases exist in mammalian cells, extracellular signal-regulated kinase (ERK), c-Jun amino-terminal kinase (JNK), and p38, each of which forms a signaling module. Each kinase module is coupled to cell surface receptors through members of the small GTP-binding protein family, including Ras, Rac, and Cdc42 $(11,12)$. Although there is "cross talk" among the pathways, the ERK pathway is most closely associated with activation of growth factor receptors, while the JNK and p38 pathways are most closely associated with activation of cytokine receptors and with cellular stress, including UV radiation. MAP kinase activation induces both c-Jun and c-Fos, which comprise, along with protein factors, the transcription factor activator protein (AP) $1(9,13,14)$.

Although the MAP kinase signaling pathways have been the subject of intense interest, studies to date have been largely restricted to cells in culture. Little is known about the extent to which MAP kinase pathways function in intact human cells in vivo. Human skin, because of its accessibility and responsiveness to UV radiation, offers unique opportunities to study signal transduction pathways in vivo.

We have reported previously that UV radiation upregulates AP-1 and induces AP-1-regulated matrix-degrading metalloproteinase genes in human skin in vivo (15). We hypothesize that this is the major mechanism by which UV damages skin connective tissue, causing premature skin aging (photoag-

1. Abbreviations used in this paper: ATF, activating transcription factor; AP, activator protein; ERK, extracellular signal-regulated kinase; GST, glutathione $S$-transferase; JNK, c-Jun amino-terminal kinase; MAP, mitogen-activated protein; MED, minimal erythema dose; $t \mathrm{RA}$, all-trans retinoic acid. 
ing) (16). We have also demonstrated that all-trans retinoic acid ( $t$ RA) applied to human skin inhibits subsequent activation of AP-1 and induction of matrix metalloproteinases by UV radiation (15). To gain insight into the mechanisms regulating matrix metalloproteinase induction and its inhibition by $t$ RA, we investigated MAP kinase signal transduction pathways that regulate AP- 1 in response to $\mathrm{UV}$ radiation in human skin in vivo.

\section{Methods}

UV irradiation and tissue procurement. Healthy adult Caucasians, with light to mild pigmentation, were irradiated with fluorescent UVB lights, as described previously (15). Wavelengths below $290 \mathrm{~nm}$ were removed by a Kodacel TA401/407 sheet (Eastman Kodak Co., Rochester, NY). The UV dose that caused barely perceptible skin reddening (minimal erythema dose, MED) was determined for each subject, $24 \mathrm{~h}$ after irradiation. Irradiated and nonirradiated skin samples were obtained from each subject by keratome or punch biopsy, as described (17). $t$ RA (Sigma Chemical Co., St. Louis, MO) and its vehicle $(70 \%$ ethanol, $30 \%$ polyethylene glycol, $0.05 \%$ 3,5-di-tera-butyl4-hydroxytoluene) were applied to skin under occlusion, 8 or $48 \mathrm{~h}$ before UV treatment. All procedures involving human subjects were approved by the University of Michigan Institutional Review Board, and all subjects provided written informed consent.

Preparation of skin supernatants for biochemical analyses. Skin samples were ground by mortar and pestle under liquid nitrogen, and homogenized in a Dounce tissue grinder in buffer containing $10 \mathrm{mM}$ Hepes, $1 \mathrm{mM}$ EDTA, $5 \mathrm{mM}$ EGTA, $10 \mathrm{mM} \mathrm{MgCl}_{2}$, $50 \mathrm{mM}$ glycerophosphate, $5 \mathrm{mM} \mathrm{NaVO}_{4}, 2 \mathrm{mM}$ DTT, $0.5 \mathrm{mM} \mathrm{PMSF}, 10 \mu \mathrm{g} / \mathrm{ml}$ aprotinin, $10 \mu \mathrm{g} / \mathrm{ml}$ leupeptin, $10 \mu \mathrm{g} / \mathrm{ml}$ pepstatin, and $0.5 \% \mathrm{NP}-40$. Homogenates were centrifuged at $14,000 \mathrm{~g}$ for $15 \mathrm{~min}$, and supernatants were collected and used for biochemical determinations described below.

EGF receptor phosphorylation. Total and tyrosine-phosphorylated EGF receptors were determined by immunoprecipitation and Western analysis, using antibodies to EGF receptors (Santa Cruz Biotechnology Inc., Santa Cruz, CA) and phosphotyrosine (Transduction Laboratories, Lexington, KY). Immunoreactive proteins were visualized by enhanced chemiluminescence detection, and quantified by laser densitometry.

Ras activation. Skin supernatants were passed immediately through PD-10 columns (Pharmacia Biotech, Piscataway, NJ) to remove free nucleotides. Proteins eluting in the void volume were collected, and $\mathrm{NaCl}$, SDS, and deoxycholate were added to $0.5 \mathrm{M}, 0.05 \%$, and $0.5 \%$ final concentrations, respectively. Ras was immunoprecipitated with rat mAb to Ras (Y13-259; Oncogene Sciences, Inc., Mineola, NY), and GDP and GTP bound to Ras were quantified as described (18).

ERK phosphorylation and activity assay. ERK1 and ERK2 in skin supernatants were immunoprecipitated with antibodies from Santa Cruz Biotechnology Inc. and assayed for enzymatic activity using myelin basic protein as substrate, as described (19). Total and phosphorylated ERK1 and ERK2 in supernatants were determined by Western analysis, using antibodies from New England Biolabs Inc. (Beverly, MA).

$J N K$ and p38 phosphorylation and c-Jun and activating transcription factor (ATF) 2 kinase assays. c-Jun and ATF-2 kinase activities in skin supernatants were determined by solid phase kinase assays, as described (20,21). JNK and p38 were immunoprecipitated with antibodies from Santa Cruz Biotechnology Inc. and assayed for activity, using glutathione $S$-transferase (GST)-c-Jun and GST-ATF-2 as substrates. p38 inhibitor SB-2030580 was kindly provided by Dr. John Lee (SmithKline Beecham Pharmaceuticals, King of Prussia, PA), and was used at $10 \mu \mathrm{M}$ concentration. Total and phosphorylated JNK and p38 in skin supernatants were determined by Western analysis, using antibodies from New England Biolabs Inc.
Electrophoretic mobility shift assay. Gel mobility shift and supershift assays were performed on nuclear extracts from human skin (22). Double-stranded oligodeoxynucleotides containing the Jun 1 (5'AGGTGGGGTGACATCATGGG) and Jun 2 (5'AGCTAGCATTACCT-CATCCC) response elements in the human c-Jun promoter, and the consensus AP-1 response element (5'CGCTTGATGAGTCAGCCGGAA), were used as probes.

Northern analysis of Jun and Fos family members. Total RNA was isolated from skin samples by guanidinium hydrochloride lysis and ultracentrifugation, as described previously (17). Northern analyses of total RNA $(40 \mu \mathrm{g} / \mathrm{lane})$ with randomly primed $\left[{ }^{32} \mathrm{P}\right] \mathrm{cDNA}$ probes for human c-Jun, Jun B, Jun D, c-Fos, Fos B, Fra-1, Fra-2, and 36B4 (a ribosomal protein used as an internal control) were performed as described (23).

Western analysis of Jun and Fos family members. Jun and Fos family member proteins (antibodies from Santa Cruz Biotechnology Inc.) and phosphorylated c-Jun (antibody from New England Biolabs Inc.) were detected in nuclear extracts from human skin by Western analysis, as described (22).

Immunohistology. Immunohistology of c-Jun was performed as described previously (24). c-Jun was detected with mouse mAb (Transduction Laboratories), using a peroxidase detection system (Vector Laboratories, Inc., Burlingame, CA). Appropriately diluted mouse IgG (Sigma Chemical Co.), used as control, yielded no detectable staining.

Statistics. Comparisons among treatment groups were made with either the paired $t$ test (two groups) or the repeated measures ANOVA (more than two groups). Multiple pairwise comparisons were made with the Tukey Studentized Range test. All $P$ values are two-tailed, and significant when $\leq 0.05$.

\section{Results}

$U V$ radiation transiently activates EGF receptors and induces sustained activation of Ras in human skin in vivo. Exposure of adult human skin to UV radiation resulted in rapid tyrosine phosphorylation of EGF receptors, as detected by an antiphosphotyrosine antibody. Tyrosine phosphorylation, a marker for EGF receptor activation (25), was detectable within 15 min after UV exposure and remained elevated for $2 \mathrm{~h}$ (Fig. 1a). EGF receptor tyrosine phosphorylation decreased to near basal levels between 2 and $8 \mathrm{~h}$ after UV exposure (Fig. $1 \mathrm{a}$ ), and remained unchanged for at least $24 \mathrm{~h}$ (data not shown). Similar results were obtained using an antibody specific for tyrosinephosphorylated EGF receptors (data not shown). UV exposure did not alter the level of total EGF receptors in human skin (Fig. $1 a$ ).

The Ras family of small GTP-binding regulatory proteins is activated by EGF and other growth factor receptors $(10,25)$. We adapted a nonisotopic method (18) to quantify the levels of activated GTP-bound Ras in human skin after exposure to UV radiation. In nonirradiated skin, $6.3 \%$ of Ras was in the activated GTP-bound state. GTP-bound Ras increased sharply (2.3-fold) from 15 to 60 min after UV exposure, and remained elevated at this level for up to $24 \mathrm{~h}$ (Fig. 1 b). Total Ras (GTP plus GDP-bound) in skin (1.0 $\pm 0.2 \mathrm{nmol}$ Ras $/ \mathrm{mg}$ protein) was not altered by UV exposure (data not shown).

$U V$ radiation stimulates $M A P$ kinases $E R K, J N K$, and $p 38$ in human skin in vivo. Ras is an upstream regulator of ERK, JNK, and p38 MAP kinase signaling pathways $(11,12)$. MAP kinases are activated by MAP kinase kinases through phosphorylation of specific threonine and tyrosine residues (26). Therefore, we examined the effect of UV radiation on both phosphorylation and activity of ERK, JNK, and p38. Phosphor- 


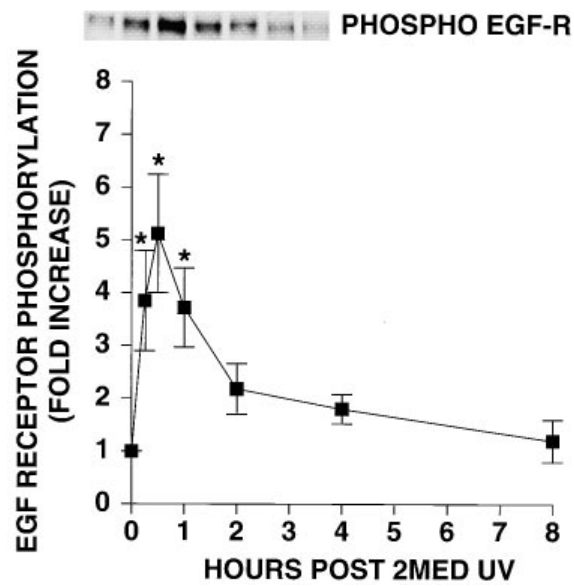

b

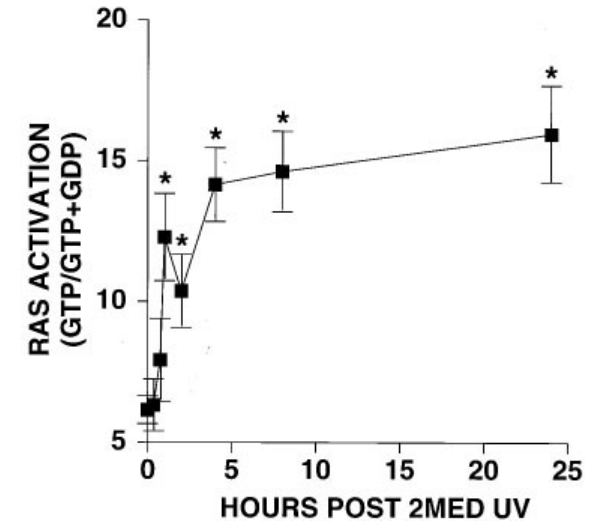

Figure 1. UV irradiation activates EGF receptors and stimulates Ras in human skin in vivo. (a) Time course of induction of EGF receptor tyrosine phosphorylation by UV irradiation. Adult human skin was obtained at the indicated times after UV irradiation $(2 M E D)$, and EGF receptors were solubilized and immunoprecipitated. Total and tyrosine-phosphorylated ( $\mathrm{PHOSPHO}$ ) EGF receptors $(E G F-R)$ in the immunoprecipitates were quantified by Western analyses, as described in Methods. Bar heights are means \pm SEM of data from 14 subjects. Skin from eight subjects was analyzed $0,0.25,0.5,1,2$, and $4 \mathrm{~h}$ after irradiation, and skin from six subjects was analyzed $0,0.5,1,2,4$, and $8 \mathrm{~h}$ after irradiation. $* P<0.05$ vs. nonirradiated control skin. Inset, Representative Western blot. (b) Time course of Ras activation by UV irradiation. Adult human skin was obtained at the indicated times after UV irradiation $(2 M E D)$, and Ras was solubilized and im-

munoprecipitated. GTP and GDP bound to immunoprecipitated Ras were quantified as described in Methods. Data are means \pm SEM from ten subjects at 0 and $4 \mathrm{~h}$ after $\mathrm{UV}$ irradiation and five subjects at the remaining times indicated. ${ }^{*} P<0.05 \mathrm{vs}$. nonirradiated control skin.

ylation of ERK1 and ERK2 increased within 30 min of UV exposure, and remained elevated for $24 \mathrm{~h}$ (Fig. $2 a$, inset). Human skin expressed considerably more ERK2 than ERK1; however, the kinetics of their phosphorylation were similar. UV radiation did not alter total ERK1 or ERK2 levels (Fig. $2 a$, inset). UV radiation also stimulated ERK1/2 activity. ERK2 activity had increased (threefold) $1 \mathrm{~h}$ after UV irradiation, remained significantly elevated for $4 \mathrm{~h}$, and returned to baseline by $16 \mathrm{~h}$ (Fig. $2 a$ ). ERK1 activity followed a pattern similar to that of ERK2 (data not shown); however, the low level of ERK1 made accurate quantitation difficult.

UV radiation also induced phosphorylation of JNK and p38. Increased phosphorylation of both JNK and p38 occurred within $1 \mathrm{~h}$ of UV exposure, remained elevated for $8 \mathrm{~h}$, and returned to near baseline levels by $24 \mathrm{~h}$ (Fig. $2 \mathrm{~b}$, inset). JNK binds to and phosphorylates transcription factors c-Jun and ATF-2 $(20,21)$, and p38 binds to and phosphorylates ATF-2 (27). Therefore, we performed solid phase kinase assays using a
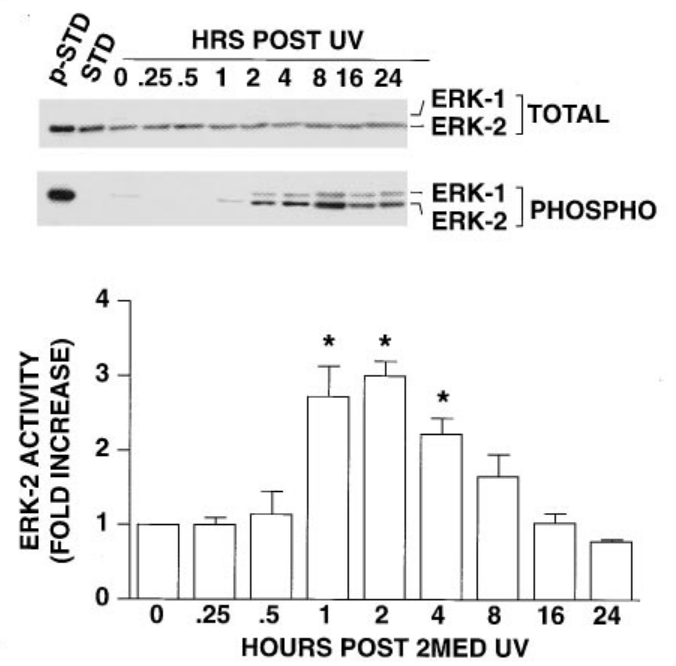

b
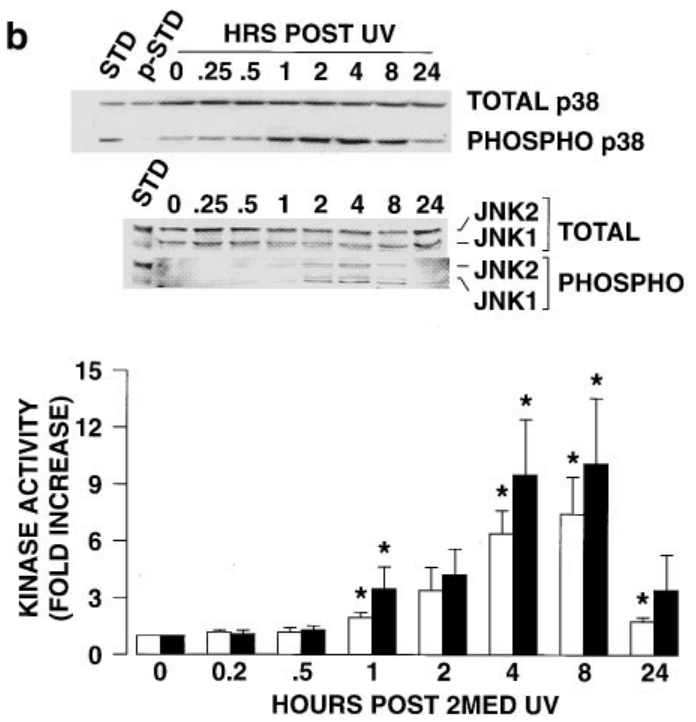

Figure 2. UV irradiation stimulates ERK, JNK, and p38 MAP kinases in human skin in vivo. $(a)$ Time course of stimulation of ERK phosphorylation and activity by UV irradiation. Skin was obtained at the indicated times after UV irradiation $(2 M E D)$ and analyzed for total and phosphorylated ERK-1 and ERK-2 by Western blot (insets), and activity, as described in Methods. Bar heights are means \pm SEM of data from 10 subjects. Skin from five subjects was analyzed $0,0.25$, $0.5,1,2$, and $4 \mathrm{~h}$ after irradiation, and skin from

five subjects was analyzed $0,4,8,16$, and $24 \mathrm{~h}$ after irradiation. ${ }^{*} P<0.05$ vs. nonirradiated control skin. (b) Time course of induction of JNK and p38 phosphorylation and activity by UV irradiation. Skin was obtained at the indicated times after UV irradiation $(2 M E D)$ and analyzed for total and phosphorylated JNK-1, JNK-2, and p38 by Western blot (insets), and ATF-2 (white bars) and c-Jun (black bars) kinase activities, as described in Methods. Bar heights are means \pm SEM of data from 10 subjects. Skin from five subjects was analyzed at $0,0.25,0.5,1,2$, and $4 \mathrm{~h}$ after irradiation, and skin from five subjects was analyzed at $0,4,8$, and $24 \mathrm{~h}$ after irradiation. $* P<0.05$ vs. nonirradiated control skin. $P H O S P H O$, Phosphorylated. $S T D$, ERK standard. $p$-STD, Phosphorylated ERK standard. 
GST-c-Jun and GST-ATF-2 as substrates to determine the effect of UV radiation on JNK and p38 activities. c-Jun and ATF-2 kinase activities were stimulated in parallel and to similar extents by UV radiation. Increased activities were detected $1 \mathrm{~h}$ after UV irradiation, which remained elevated (sevenfold or greater) for at least $8 \mathrm{~h}$, and returned to near baseline levels by $24 \mathrm{~h}$ (Fig. $2 b$ ).

$U V$-induced $J N K$ and p38 bind to and phosphorylate c-Jun and $A T F-2$. We next performed pulldown studies to verify the identity of UV radiation-induced kinases that bound to and phosphorylated c-Jun and ATF-2 in human skin. As expected, JNK1 and JNK2 bound to both GST-c-Jun and GST-ATF-2. No binding of these kinases to GST alone was detected (data not shown). In addition, p38 bound to both ATF-2 and c-Jun (Fig. $3 a$ ). This finding was unexpected, since it has been reported that p38 does not bind to or phosphorylate c-Jun (27). Therefore, we conducted immune complex assays to determine whether p38 phosphorylated c-Jun in human skin. p38 was immunoprecipitated from nonirradiated and UV-irradiated skin extracts, and assayed to determine its ability to phosphorylate GST-c-Jun and GST-ATF-2. Phosphorylation of either substrate by p38 immune complexes from nonirradiated skin was minimal. In contrast, p38 immune complexes from UV-irradiated skin phosphorylated markedly both c-Jun and ATF-2 (Fig. $3 b$ ). Phosphorylation of ATF-2 by p38 was 2.5fold greater $(n=6)$ than phosphorylation of c-Jun. Phosphorylation of both substrates was blocked substantially by the p38 inhibitor SB-203580 (Fig. 3 b), confirming that p38 immunoprecipitated from human skin phosphorylates both c-Jun and ATF-2. Immune complex kinase assays also demonstrated that UV radiation induced $\mathrm{JNK} 1 / 2$-catalyzed phosphorylation of both c-Jun and ATF-2 in human skin (data not shown). SB203580 did not inhibit JNK1/2-catalyzed c-Jun or ATF-2 phosphorylation, confirming that SB-203580 specifically inhibits p38 (data not shown).

$U V$ radiation induces c-Jun and ATF-2 binding to the c-Jun promoter. Phosphorylation of c-Jun and ATF-2 by JNK and p38 stimulates their transactivation activities but does not appear to alter their binding to DNA directly. Therefore, we examined whether UV radiation alters DNA binding of c-Jun or
ATF-2 in human skin. For these experiments, we used the Jun 1 and Jun 2 response elements in the c-Jun promoter as probes. These elements have been shown to bind c-Jun/ATF-2 heterodimers, stimulating c-Jun gene transcription $(28,29)$. Gel shift analyses of nuclear extracts from nonirradiated human skin revealed specific retarded complexes bound to both the Jun 1 and Jun 2 elements (Fig. 4, $a$ and $b$ ). Supershifts indicated that these complexes contained low levels of c-Jun and ATF-2. Within $2 \mathrm{~h}$ of UV irradiation, the levels of c-Jun and ATF-2 in the retarded complexes bound to Jun 1 and Jun 2 had increased (Fig. 4, $a$ and $b$ ).

$U V$ radiation induces $c$-Jun and Jun $B m R N A$ and protein in human skin in vivo. Activation of the ERK and JNK/p38 pathways results in induction of c-Fos and c-Jun in cultured cells $(9,14)$. Therefore, we examined UV induction of Jun and Fos family members in human skin in vivo. Nonirradiated skin expressed mRNA and proteins for all Jun family members, including c-Jun, Jun B, and Jun D (data not shown). UV irradiation induced c-Jun and Jun B mRNAs (Fig. $5 a$ ) and proteins (Fig. $5 \mathrm{~b}$ ). In contrast, Jun D was expressed constitutively in skin, but not induced by UV radiation (data not shown). Maximal induction of c-Jun mRNA (sixfold) and protein (fourfold) occurred 2 and $8 \mathrm{~h}$ after UV irradiation, respectively (Fig. 5). UV induction of Jun B protein lagged behind that of c-Jun, peaking $8 \mathrm{~h}$ after UV irradiation. c-Jun and Jun B mRNAs and proteins remained elevated for at least $24 \mathrm{~h}$ (Fig. 5, $a$ and $b$ ). In nonirradiated human skin, Fos family members Fos B, Fra-1, and Fra-2 mRNAs and proteins were low or undetectable. In contrast, c-Fos mRNA and protein were readily detectable (data not shown). UV radiation did not alter c-Fos mRNA or protein levels, or Fra-2 protein levels (Fra-2 mRNA was undetectable). Fos B protein was not detectable. UV radiation did, however, induce Fra-1. Fra-1 mRNA and protein were maximally induced (threefold) $8 \mathrm{~h}$ after exposure and remained elevated for at least $24 \mathrm{~h}$ (data not shown).

$U V$ radiation induces c-Jun phosphorylation and $A P-1$ $D N A$ binding in human skin in vivo. The above data demonstrate that UV radiation activates c-Jun kinases JNK and p38, and induces c-Jun expression. Therefore, we examined whether endogenous c-Jun is phosphorylated in nonirradiated and UV-
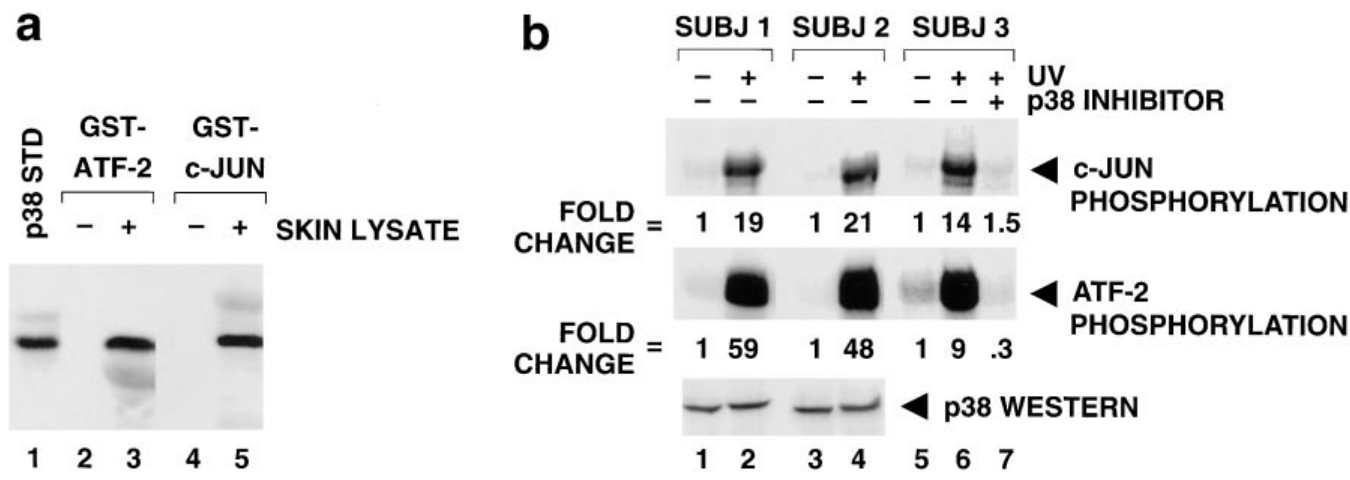

Figure 3. p38 MAP kinase from human skin binds to and phosphorylates both c-Jun and ATF-2. (a) Soluble extracts from adult human skin were incubated with ATF-2, or c-Jun bound to GST-agarose beads. The beads were washed, and bound proteins were eluted with detergent-containing buffer. Eluted proteins were subjected to Western analysis with antibodies to p38. Lane 1 , p38 standard

(STD); lane 2, GST-ATF-2 beads without skin lysate; lane 3, GST-ATF-2 beads incubated with skin lysate; lane 4, GST-c-Jun beads without skin lysate; lane 5, GST-c-Jun beads incubated with skin lysate. Results are representative of three experiments. $(b)$ p38 in soluble extracts from nonirradiated ( $-U V$, lanes 1,3 and 5) and $U V$-irradiated ( $+U V, 4 \mathrm{~h}$ after UV irradiation, lanes 2, 4, 6 and 7) human skin was immunoprecipitated and assayed for its ability to phosphorylate c-Jun (top) and ATF-2 (middle), as described in Methods. Representative results from three subjects $(S U B J)$ are displayed. Bottom, Western analysis of p38 in soluble extracts from two subjects. Lane 7, Inhibition of UV radiation-induced p38 activity by the specific p38 inhibitor, SB-203580. 
a

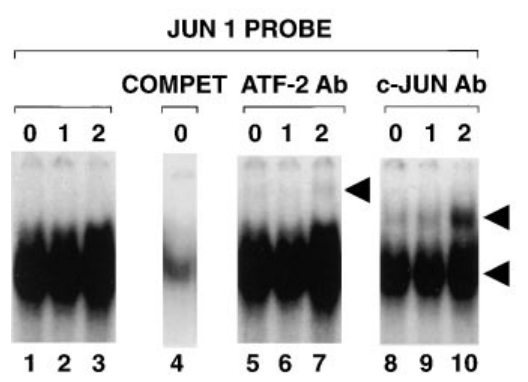

b

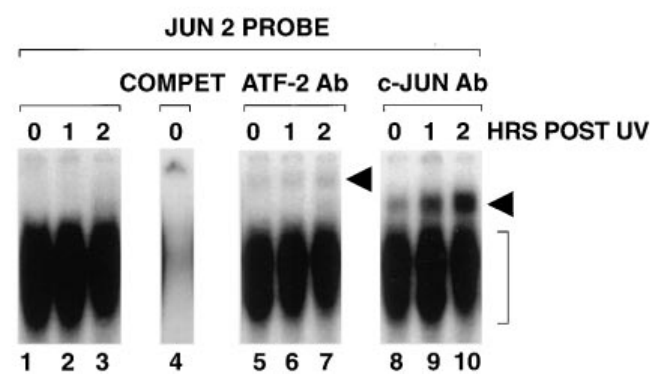

Figure 4. UV irradiation induces c-Jun and ATF-2 DNA binding to Jun 1 and Jun 2 response elements. Nuclear extracts were prepared from nonirradiated and UV-irradiated human skin ( 1 and $2 \mathrm{~h}$ after irradiation) and analyzed by gel mobility retardation assays for binding to Jun $1(a)$ and Jun $2(b)$ response elements, as described in Methods. Bottom arrowhead to the right of lane 10 in $a$ and bracket to the right of lane 10 in $b$ indicate positions of specific retarded complexes ( $a$ and $b$, lanes 1-3). $a$ and $b$, lane 4 , Reduced binding in the presence of 100-fold excess unlabeled probe (COMPET). Arrowheads to the right of lane 7 in $a$ and $b$ indicate position of ATF-2-containing complexes supershifted by ATF-2 antibody ( $a$ and $b$, lanes 5-7). Top arrowhead to the right of lane 10 in $a$ and arrowhead to the right of lane 10 in $b$ indicate positions of c-Jun-containing complexes supershifted by c-Jun antibody. Results are representative of five experiments. $A b$, Antibody.

irradiated human skin, using an antibody specific for phosphorylated serine 63 , one of two serines phosphorylated by JNK. Phosphorylation of endogenous c-Jun protein was increased $1 \mathrm{~h}$ after UV irradiation, and was further increased at 4-16 h, during which time total c-Jun protein also accumulated (Fig. 6 $a$ ). These data are consistent with the time courses of induction of c-Jun kinase activities (Fig. $2 b$ ) and c-Jun protein (Fig. $5 b$ ) by UV radiation.

Phosphorylation of c-Jun by both JNK and p38 stimulates its transactivation activity (21). c-Jun binds to AP-1 response elements in target gene promoters as homodimers or heterodimers with other Jun or Fos family members. Therefore, we examined whether Jun and Fos family members bind to DNA in nonirradiated and UV-irradiated human skin. Nuclear extracts from nonirradiated skin formed specific retarded complexes with a consensus AP-1 response element (Fig. 6b). Antibody supershifts revealed that these complexes contained c-Jun, Jun B, Jun D, c-Fos, Fra-1, and Fra-2, all the Jun and Fos family members expressed in skin (data not shown). UV radiation increased the intensity of the AP-1 retarded complexes, and increased the relative level of c-Jun in them (Fig. 6 $b$ ). As expected, levels of c-Fos were not altered in response to UV radiation (Fig. $6 b$ ).
tRA inhibits $U V$ radiation-induced $c$-Jun and Jun B protein induction. The above data demonstrate that UV radiation induces increased DNA binding of AP-1 complexes that are enriched in c-Jun but not other Jun or Fos family members. Induction of c-Jun protein and its phosphorylation by JNK and p38 likely lead to functional activation of AP-1 complexes that stimulate transcription in target genes in human skin in vivo. We have shown previously that UV radiation induces AP1-regulated matrix metalloproteinase genes (collagenase, 92$\mathrm{kD}$ gelatinase, and stromelysin) in human skin in vivo, and that this induction is inhibited by pretreatment of skin with $t \mathrm{RA}$ for $48 \mathrm{~h}$ (15). Therefore, we examined the effect of $t \mathrm{RA}$ on UV induction of c-Jun mRNA and protein, and c-Jun-containing AP-1 complexes.

Pretreatment of human skin with $0.1 \% t \mathrm{RA}$ for $48 \mathrm{~h}$ had no effect on basal c-Jun mRNA or protein levels, and did not inhibit UV induction of c-Jun mRNA (Fig. $7 a$ ). In contrast, UV induction of c-Jun protein was inhibited $70 \%$ by pretreatment with $t$ RA (Fig. $7 a$ ). This inhibition was observed at the onset of accumulation of c-Jun protein, $4 \mathrm{~h}$ after UV exposure, and later, at $16 \mathrm{~h}$ after $\mathrm{UV}$ radiation (Fig. $7 \mathrm{~b}$ ), indicating that $t \mathrm{RA}$ blocked induction of c-Jun protein instead of altering the kinetics of c-Jun protein accumulation. Pretreatment with $t \mathrm{RA}$

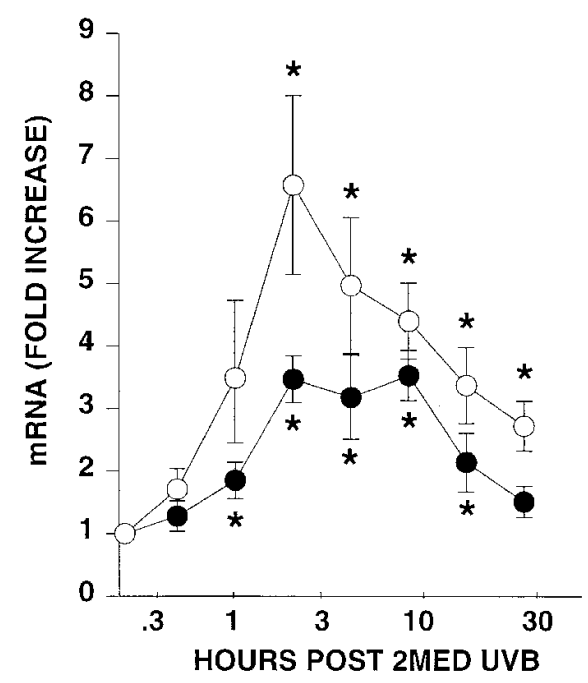

b

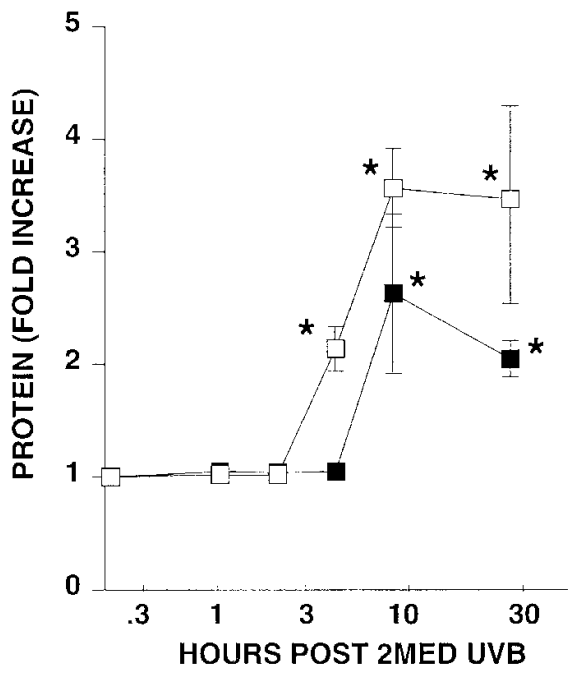

Figure 5. UV irradiation induces c-Jun and Jun B in human skin in vivo. Adult human skin was obtained at the indicated times after UV irradiation (2MED). Total RNA or nuclear extracts were prepared for Northern or Western analyses, respectively, of c-Jun and Jun B. (a) Time course of c-Jun and Jun B mRNA induction by UV radiation in human skin in vivo. (b) Time course of c-Jun and Jun B protein induction by UV radiation in human skin in vivo. c-Jun mRNA (O); Jun B mRNA (๑); c-Jun protein ( $\square$ ); Jun B protein (ם). Data are expressed as fold increase relative to nonirradiated controls. Data are means \pm SEM from 17 subjects $(m R N A)$ or eight subjects (PROTEIN). $* P<0.05$ vs. nonirradiated control skin. 


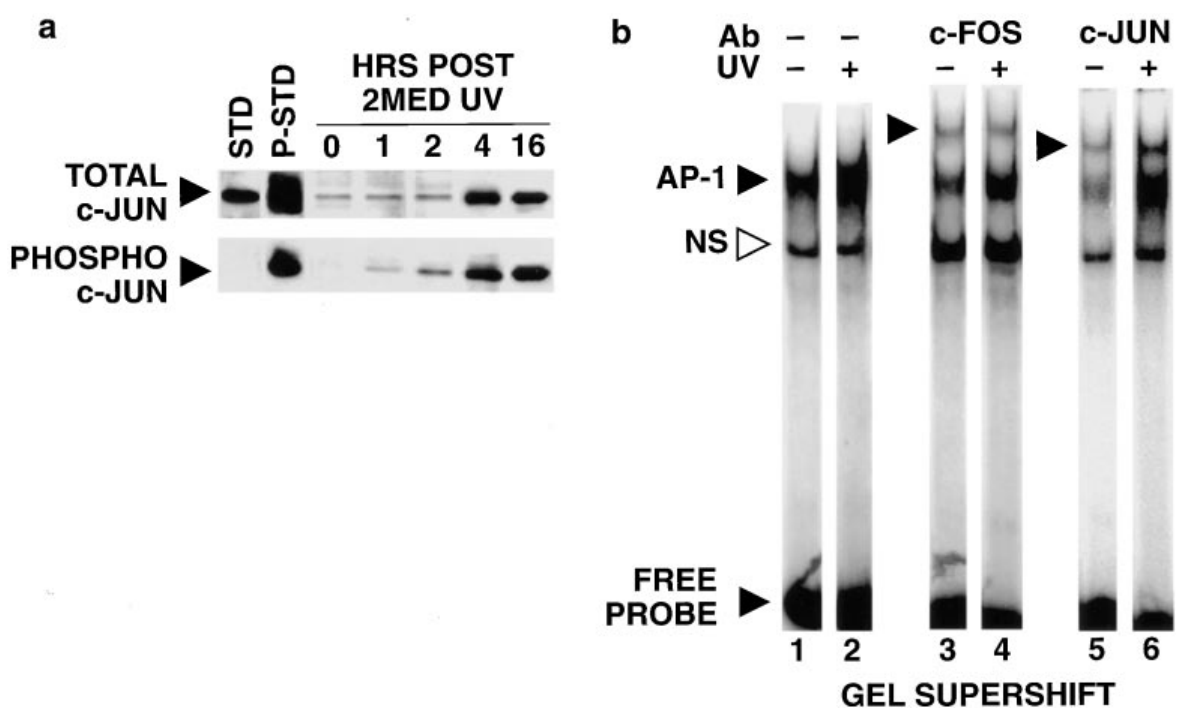

Figure 6. UV irradiation induces aminoterminal phosphorylation of c-Jun and AP-1 complexes enriched in c-Jun in human skin in vivo. (a) Nuclear extracts were prepared from human skin at the indicated times after UV irradiation (2MED) and analyzed for total and amino-terminal-phosphorylated (serine 63) c-Jun by Western analysis. $S T D$, Nonphosphorylated c-Jun standard protein. $P$-STD, Phosphorylated c-Jun standard protein. Results are representative of six experiments. (b) AP-1 DNA binding in nuclear extracts from nonirradiated (lanes 1,3, and 5) and UV-irradiated (4 $\mathrm{h}$ after UV irradiation, lanes 2, 4, and 6 ) human skin was determined by gel mobility retardation assay. Black arrowhead (AP-1), Position of specific AP-1 complexes in all lanes. White arrowhead $(N S)$, Position of a nonspecific band in all lanes. Black arrowhead to the left of lane 3 indicates position of c-Fos-containing complexes in lanes 3 and 4 supershifted by antibody $(A b)$ to c-Fos. Black arrowhead to the left of lane 5 indicates position of c-Jun-containing complexes in lanes 5 and 6 supershifted with antibody to c-Jun. Data are representative of six experiments. PHOSPHO, Phosphorylated.

also inhibited induction of AP-1 complexes by UV radiation (Fig. $7 c$ ), a finding consistent with increased formation of AP-1 complexes being dependent on elevated c-Jun protein levels. Treatment of skin with $t$ RA for $48 \mathrm{~h}$ also inhibited UV induction of Jun B protein ( $69 \pm 9 \%$ inhibition, $n=7)$.

Interestingly, pretreatment with $t \mathrm{RA}$ for $8 \mathrm{~h}$ had no effect on UV induction of c-Jun or Jun B protein, AP-1 DNA bind- ing, c-Jun-containing AP-1 complexes, or matrix metalloproteinases (data not shown). This finding is in contrast to the effects of pretreatment with $t \mathrm{RA}$ for $48 \mathrm{~h}$, which, as described above, inhibited all of these UV-induced responses.

Immunohistology revealed that UV radiation induced c-Jun protein throughout the upper (epidermis) and lower (dermis) compartments of skin (Fig. 8, $a$ and $b$ ). Pretreatment of human a
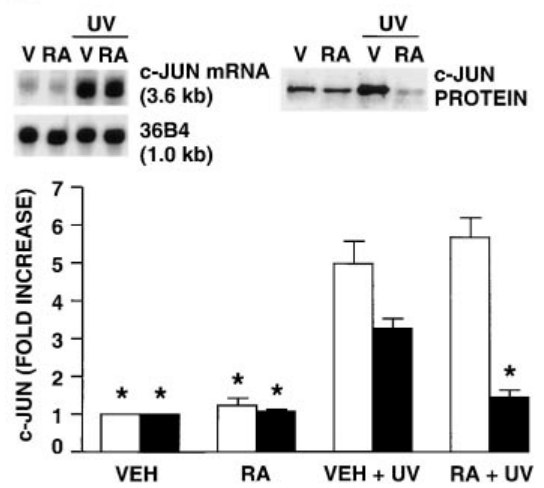

b

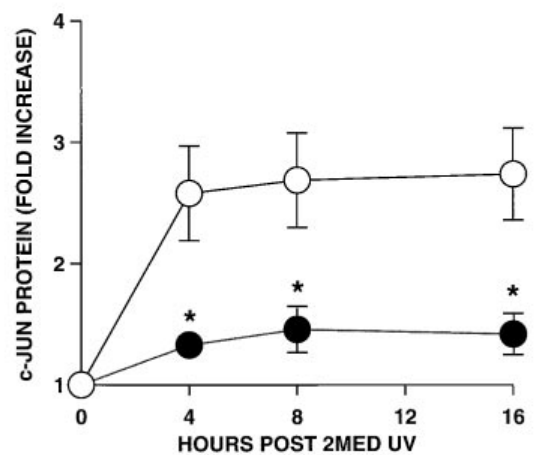

C

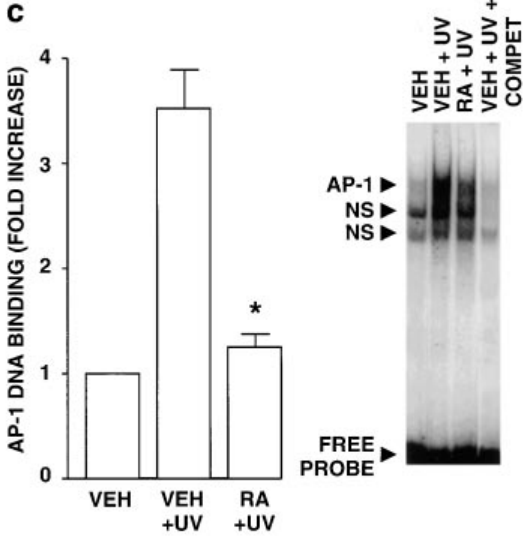

Figure 7. $t$ RA pretreatment inhibits UV induction of c-Jun protein and AP-1 in human skin in vivo. (a) Adult human skin was pretreated on two sites with vehicle $(V E H)$ and on two sites with $0.1 \% t \mathrm{RA}(R A)$ for $48 \mathrm{~h}$. One vehicle-treated site and one $t$ RA-treated site were UV-irradiated, and skin was obtained from all sites $8 \mathrm{~h}$ after UV irradiation. c-Jun mRNA was quantified by Northern analysis (white bars), and c-Jun protein, in nuclear extracts, was quantified by Western analysis (black bars). Insets, Representative Northern and Western blots. $V$, Vehicle. $R A, t$ RA. 36B4 mRNA served as an internal control for quantitation of Northern analyses. Bar heights are means \pm SEM of six subjects for Northern analyses and eight subjects for Western analyses. ${ }^{*} P<0.05$ vs. vehicle-treated, UV-irradiated skin. $(b)$ Subjects were pretreated with vehicle $(O)$ or $0.1 \% t \mathrm{RA}(\bullet)$ for $48 \mathrm{~h}$ before UV irradiation $(2 M E D)$. Skin was obtained at $4(n=4), 8(n=7)$, and $16(n=6) \mathrm{h}$ after UV irradiation, and c-Jun protein in nuclear extracts was quantified by Western analyses. Results are means \pm SEM. $* P<0.05$ vs. vehicle-treated, UV-irradiated skin. $(c)$ Vehicle $(V E H)$ or $0.1 \% t$ RA $(R A)$ were applied to human skin $48 \mathrm{~h}$ before UV irradiation. Skin was obtained $8 \mathrm{~h}$ after UV irradiation, and nuclear extracts were prepared. AP-1 DNA binding was determined by gel mobility shift analysis as described in Methods. Bars are means \pm SEM of four experiments. $* P<0.05$ vs. vehicle-treated, UV-irradiated skin. Right, A representative gel. In the far right lane, a 100-fold excess of unlabeled probe (COMPET) was added. Black arrowhead (AP-1), Position of specific AP-1 complexes in all lanes. Black arrowhead (NS), Position of a nonspecific band in all lanes. 
$a$

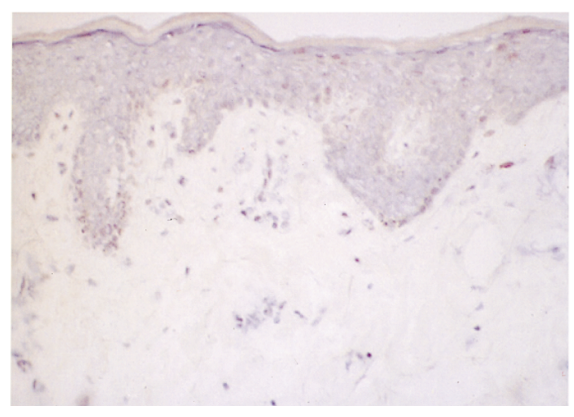

$b$

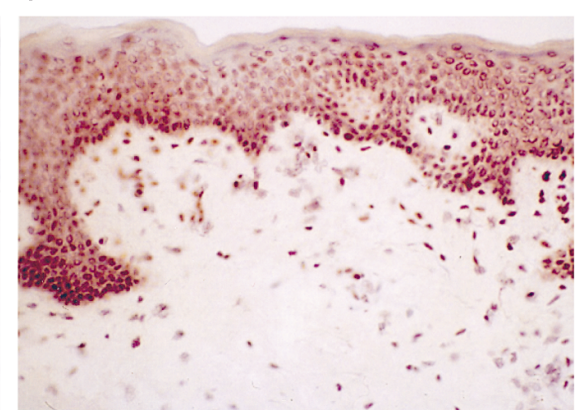

$c$

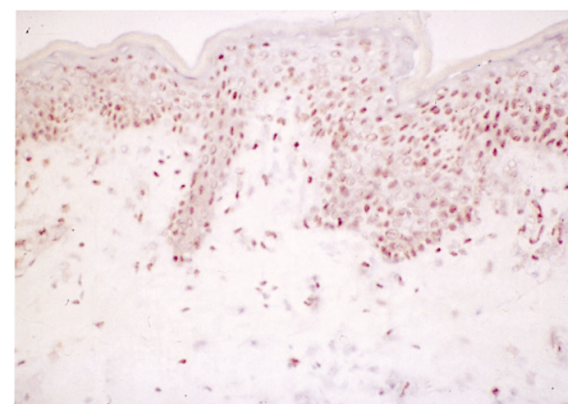

Figure 8. Immunohistological localization of c-Jun protein induction by UV irradiation and inhibition by $t$ RA. Adult human skin was pretreated with vehicle or $0.1 \% t$ RA for $48 \mathrm{~h}$ before UV irradiation. Skin was obtained $8 \mathrm{~h}$ after UV irradiation, and c-Jun protein was localized by immunohistology. (a) Vehicle-treated, nonirradiated skin; $(b)$ vehicle-treated, UV-irradiated skin; (c) $t$ RA-pretreated, UV-irradiated skin. Results are representative of six experiments.

skin in vivo with $t$ RA for $48 \mathrm{~h}$ before $\mathrm{UV}$ irradiation reduced substantially c-Jun induction compared with vehicle-treated, UV-irradiated skin in both the epidermis and dermis (Fig. 8c).

\section{Discussion}

The data presented above demonstrate that exposure of human skin in vivo to low levels of UV radiation activates MAP kinase signal transduction pathways. These pathways mediate responses to growth factors, cytokines, and stress (including UV radiation) in cultured cells $(9,10,14,25)$. UV radiationinduced signal transduction in human skin in vivo exhibits novel features that have not been observed in cultured cells, including phosphorylation of c-Jun by p38 MAP kinase, constitutive expression and lack of induction of c-Fos, and inhibition of c-Jun protein induction by $t \mathrm{RA}$.

UV radiation rapidly activated EGF receptors in human skin. In cultured cells, including skin cells, EGF receptor activation is coupled to activation of Ras, which in turn activates ERK and, to a lesser extent, JNK MAP kinase pathways (8-10, 25). In human skin, UV radiation also activated Ras, ERK, and JNK, and the kinetics of this activation were consistent with it being the result of EGF receptor activation. In cultured cells, UV radiation activates several types of cell surface receptors, which have in common the requirement for dimerization to initiate signal transduction (4-6). Human skin expresses several members of this class of receptors. It is likely that activation of these receptors, in addition to EGF receptors, contributed to the observed stimulation of MAP kinase pathways by UV radiation. Activation of multiple types of receptors may account for the sustained activation of Ras and MAP kinases, given that activation of EGF receptors in human skin by UV radiation was relatively transient. In addition, Ras displayed two peaks of activation: an initial peak at $1 \mathrm{~h}$, and a second, sustained peak beginning at $4 \mathrm{~h}$ after UV exposure. This observation may reflect differences in the kinetics of activation of different types of Ras-coupled receptors. It is also possible that the second peak may reflect release of growth factors and cytokines by skin cells, which are a rich source of these factors, and subsequent receptor activation through autocrine and paracrine mechanisms.

The effect of UV irradiation on stress-activated MAP kinase activities in human skin was assessed by measuring phos- phorylation of c-Jun and ATF-2 in vitro. UV radiation stimulated phosphorylation of both substrates to similar extents. Pulldown studies revealed that JNK1/2 and p38 bound to both c-Jun and ATF-2. This finding suggested that both JNK and p38 phosphorylated both substrates, a possibility that was confirmed by immune complex kinase assays. In cultured cells, phosphorylation of c-Jun by JNK1/2, and phosphorylation of ATF-2 by JNK1/2 and p38 have been observed (20, 21, 30, 31). However, phosphorylation of c-Jun by p38 has not been reported, and one study concluded that p38 does not bind to or phosphorylate c-Jun (27). The explanation for the difference in findings between that study and ours is unknown. However, the discrepancy may be due to differences in experimental systems, since the prior study used transformed cell lines. Nevertheless, in UV-irradiated human skin, p38 is capable of phosphorylating c-Jun as well as ATF-2, allowing for phosphorylation of both c-Jun and ATF-2 in response to stimuli that preferentially activate either p38 or JNK.

In human skin, c-Fos and Jun D proteins were expressed constitutively, and not induced by UV irradiation. Jun D is also expressed constitutively in many types of cultured cells (32). c-Fos, however, is not. c-Fos is expressed typically at low levels in cultured cells (albeit rendered quiescent by removal of serum or growth factors), and induced by addition of factors that stimulate growth or differentiation (33). This induction occurs through transcriptional mechanisms that have been studied extensively in vitro. Immunohistology revealed that c-Fos was expressed in all of the cell layers throughout adult human skin (data not shown). Constitutive expression of c-Fos may reflect its role in normal skin cell proliferation and differentiation, which occur continuously. However, c-Fos must heterodimerize with a Jun family member to form an active AP-1 complex. Our data indicate that Jun D may be the predominant heterodimeric partner for c-Fos in human skin under normal physiological conditions.

In contrast to c-Fos and Jun D, c-Jun, Jun B, and Fra-1 proteins were induced by UV radiation in human skin. c-Jun induction occurred at $4 \mathrm{~h}$ after UV exposure, preceding that of Jun B and Fra-1 by $4 \mathrm{~h}$. Amino-terminal phosphorylation of c-Jun, catalyzed by JNK and p38, was also elevated at $4 \mathrm{~h}$, suggesting that accumulating c-Jun protein was phosphorylated and activated. Gel mobility shift analyses revealed that AP-1 complexes were enriched in c-Jun protein 4-8 h after UV irra- 
diation. This increase correlates with induced gene expression of the matrix metalloproteinases, collagenase, $92-\mathrm{kD}$ gelatinase, and stromelysin, in human skin (15). These genes are strongly positively regulated by AP-1 complexes composed of c-Jun/ c-Fos heterodimers (34). In contrast, Jun B and Fra-1 are negative regulators of c-Jun/c-Fos heterodimers (35), and their induction in human skin at later times after UV irradiation may therefore serve to restrict AP-1 activity.

$t \mathrm{RA}$ is an important regulator of cellular homeostasis in many tissues, including human skin. The actions of $t \mathrm{RA}$ are mediated by nuclear retinoid receptors that are ligand-activated transcription factors (36). In mouse skin and a variety of cultured cells, $t$ RA inhibits many of the effects of phorbol esters. This inhibition occurs principally through antagonism of AP-1 $(37,38)$. We have reported that $t$ RA blocks increases in AP-1 DNA binding and induction of matrix metalloproteinases by UV radiation in human skin in vivo. The data presented above indicate that this inhibition of AP-1 may occur through a novel mechanism distinct from previously described transrepression (37-39), involving inhibition of c-Jun protein induction. Expression of c-Jun is regulated by both transcriptional and posttranscriptional mechanisms. c-Jun transcription is induced, at least in part, through activation and binding of c-Jun/ATF-2 heterodimers to two well-characterized response elements in the c-Jun promoter $(28,29)$. $t$ RA did not alter UV induction of c-Jun mRNA. In contrast, $t$ RA prevented UV induction of c-Jun protein. This result could have occurred either through inhibition of c-Jun translation and/or accelerated degradation of c-Jun. At present, our data do not allow us to distinguish between these two mechanisms, nor do they exclude other mechanisms by which $t$ RA may prevent UV induction of metalloproteinases.

Although little is known about regulation of c-Jun translation, c-Jun breakdown is known to occur through a proteasome-dependent pathway (40). Recent evidence indicates that phosphorylation of c-Jun by JNK reduces its susceptibility to proteasome attack, significantly increasing its half-life (41). Such a mechanism could partly explain the sustained elevation of c-Jun protein (for at least $24 \mathrm{~h}$ ) induced by UV radiation in human skin, since UV radiation stimulated c-Jun phosphorylation. If so, then $t$ RA might stimulate c-Jun dephosphorylation, promoting its degradation. Interestingly, inhibition of c-Jun protein induction required prolonged pretreatment $(>8 \mathrm{~h})$ with $t$ RA before UV irradiation. Under the experimental conditions we used, $t$ RA penetrates skin and fully activates nuclear retinoid receptors and the target gene cellular retinoic acidbinding protein II (CRABP II), within $6 \mathrm{~h}$ (42). Therefore, it is likely that the mechanism by which $t$ RA inhibits c-Jun protein induction involves new gene expression, presumably mediated directly or indirectly by nuclear retinoid receptors.

Understanding the molecular basis for $t$ RA's inhibition of c-Jun induction may provide us with new insights and therapeutic targets for prevention of skin damage by UV radiation, and prevention of other pathological conditions in which upregulation of AP-1 plays a prominent role, such as cancer and inflammatory diseases.

\section{Acknowledgments}

The authors acknowledge Carolyn Petersen and Suzan Rehbine for tissue procurement, Ted Hamilton for statistical analyses, Laura
VanGoor for preparation of figures, and Anne Chapple for editorial assistance.

This study was supported in part by the Dermatology Foundation (to S. Kang), by the Babcock Endowment for Dermatological Research, and by a grant from Johnson \& Johnson Corporation.

\section{References}

1. Shea, R., and J.A. Parrish. 1991. Nonionizing radiation and the skin. In Physiology, Biochemistry, and Molecular Biology of the Skin. Vol. II. L.A Goldsmith, editor. Oxford University Press, New York. 910-927.

2. Lavker, R.M., D.A. Veres, C.J. Irwin, and K.H. Kaidbey. 1995. Quantitative assessment of cumulative damage from repetitive exposures to suberythemogenic doses of UVA in human skin. Photochem. Photobiol. 62:348-352.

3. Warmuth, I., Y. Harth, M.S. Matsui, N. Wang, and V.A. DeLeo. 1994. Ultraviolet radiation induces phosphorylation of the epidermal growth factor receptor. Cancer Res. 54:374-376.

4. Sachsenmaier, C., A. Radler-Pohl, R. Zinck, A. Nordheim, P. Herrlich, and H.J. Rahmsdorf. 1994. Involvement of growth factor receptors in the mammalian UVC response. Cell. 78:963-972.

5. Rosette, C., and M. Karin. 1996. Ultraviolet light and osmotic stress: activation of the JNK cascade through multiple growth factor and cytokine receptors. Science. 274:1194-1197.

6. Huang, R.P., J.X. Wu, Y. Fan, and E.D. Adamson. 1996. UV activates growth factor receptors via reactive oxygen intermediates. J. Cell Biol. 133:211-220.

7. Bender, K., C. Blattner, A. Knebel, M. Iordanov, P. Herrlich, and H.L. Rahmsdorf. 1997. UV-induced signal transduction. J. Photochem. Photobiol. 37:1-17.

8. Davis, R.J. 1993. The mitogen-activated protein kinase signal transduction pathway. J. Biol. Chem. 268:14553-14556.

9. Karin, M., and T. Hunter. 1995. Transcriptional control by protein phosphorylation: signal transmission from the cell surface to the nucleus. Curr. Biol. 5:747-757.

10. Denhardt, D.T. 1996. Signal-transducing protein phosphorylation cascades mediated by Ras/Rho proteins in the mammalian cell: the potential for multiplex signalling. Biochem. J. 318:729-747.

11. Coso, O.A., M. Chiariello, J.C. Yu, H. Teramoto, P. Crespo, N. Xu, T. Miki, and J.S. Gutkind. 1995. The small GTP-binding proteins Rac1 and Cdc42 regulate the activity of the JNK/SAPK signaling pathway. Cell. 81:1137-1146.

12. Minden, A., A. Lin, F.X. Claret, A. Abo, and M. Karin. 1995. Selective activation of the JNK signaling cascade and c-Jun transcriptional activity by the small GTPases Rac and Cdc42Hs. Cell. 81:1147-1157.

13. Claret, F.X., M. Hibi, S. Dhut, T. Toda, and M. Karin. 1996. A new group of conserved coactivators that increase the specificity of AP-1 transcription factors. Nature. 383:453-457.

14. Whitmarsh, A.J., and R.J. Davis. 1996. Transcription factor AP-1 regulation by mitogen-activated protein kinase signal transduction pathways. $J$. Mol. Med. 74:589-607.

15. Fisher, G.J., S.C. Datta, H.S. Talwar, Z.Q. Wang, J. Varani, S. Kang, and J.J. Voorhees. 1996. Molecular basis of sun-induced premature skin ageing and retinoid antagonism. Nature. 379:335-339.

16. Fisher, G.J., Z.Q. Wang, S.C. Datta, J. Varani, S. Kang, and J.J. Voorhees. 1997. Pathophysiology of premature skin aging induced by ultraviolet light. N. Engl. J. Med. 337:1419-1428.

17. Fisher, G.J., J. Esmann, C.E. Griffiths, H.S. Talwar, E.A. Duell, C. Hammerberg, J.T. Elder, L.J. Finkel, G.D. Karabin, and B.J. Nickoloff. 1991. Cellular, immunologic and biochemical characterization of topical retinoic acid-treated human skin. J. Investig. Dermatol. 96:699-707.

18. Scheele, J.S., J.M. Rhee, and G.R. Boss. 1995. Determination of absolute amounts of GDP and GTP bound to Ras in mammalian cells: comparison of parental and Ras-overproducing NIH $3 \mathrm{~T} 3$ fibroblasts. Proc. Natl. Acad. Sci. USA. 92:1097-1100.

19. Weber, J.D., D.M. Raben, P.J. Phillips, and J.J. Baldassare. 1997. Sustained activation of extracellular-signal regulated kinase 1 (ERK1) is required for the continued expression of cyclin D1 in G1 phase. Biochem. J. 326:61-68.

20. Gupta, S., D. Campbell, B. Derijard, and R.J. Davis. 1995. Transcription factor ATF2 regulation by the JNK signal transduction pathway. Science. 267: 389-393.

21. Hibi, M., A. Lin, T. Smeal, A. Minden, and M. Karin. 1993. Identification of an oncoprotein- and UV-responsive protein kinase that binds and potentiates the c-Jun activation domain. Genes Dev. 7:2135-2148.

22. Fisher, G.J., H.S. Talwar, J.H. Xiao, S.C. Datta, A.P. Reddy, M.P. Gaub, C. Rochette-Egly, P. Chambon, and J.J. Voorhees. 1994. Immunological identification and functional quantitation of retinoic acid and retinoid $\mathrm{X}$ receptor proteins in human skin. J. Biol. Chem. 269:20629-20635.

23. Fisher, G.J., A.P. Reddy, S.C. Datta, S. Kang, J.Y. Yi, P. Chambon, and J.J. Voorhees. 1995. All-trans retinoic acid induces cellular retinol-binding protein in human skin in vivo. J. Investig. Dermatol. 105:80-86.

24. Talwar, H.S., C.E. Griffiths, G.J. Fisher, T.A. Hamilton, and J.J. Voorhees. 1995. Reduced type I and type III procollagens in photodamaged 
adult human skin. J. Investig. Dermatol. 105:285-290.

25. Ullrich, A., and J. Schlessinger. 1990. Signal transduction by receptors with tyrosine kinase activity. Cell. 61:203-212.

26. Zheng, C.F., and K.L. Guan. 1993. Properties of MEKs, the kinases that phosphorylate and activate the extracellular signal-regulated kinases. J. Biol. Chem. 268:23933-23939.

27. Raingeaud, J., S. Gupta, J.S. Rogers, M. Dickens, J. Han, R.J. Ulevitch, and R.J. Davis. 1995. Pro-inflammatory cytokines and environmental stress cause p38 mitogen-activated protein kinase activation by dual phosphorylation on tyrosine and threonine. J. Biol. Chem. 270:7420-7426.

28. Rozek, D., and G.P. Pfeifer. 1993. In vivo protein-DNA interactions at the c-jun promoter: preformed complexes mediate the UV response. Mol. Cell. Biol. 13:5490-5499.

29. van Dam, H., D. Wilhelm, I. Herr, A. Steffen, P. Herrlich, and P. Angel. 1995. ATF-2 is preferentially activated by stress-activated protein kinases to mediate c-jun induction in response to genotoxic agents. EMBO (Eur. Mol. Biol. Organ.) J. 14:1798-1811.

30. Minden, A., A. Lin, T. Smeal, B. Derijard, M. Cobb, R. Davis, and M. Karin. 1994. c-Jun N-terminal phosphorylation correlates with activation of the JNK subgroup but not the ERK subgroup of mitogen-activated protein kinases. Mol. Cell. Biol. 14:6683-6688.

31. Johnson, N.L., A.M. Gardner, K.M. Diener, C.A. Lange-Carter, J. Gleavy, M.B. Jarpe, A. Minden, M. Karin, L.I. Zon, and G.L. Johnson. 1996. Signal transduction pathways regulated by mitogen-activated/extracellular response kinase kinase kinase induce cell death. J. Biol. Chem. 271:3229-3237.

32. Pfarr, C.M., F. Mechta, G. Spyrou, D. Lallemand, S. Carillo, and M. Yaniv. 1994. Mouse JunD negatively regulates fibroblast growth and antagonizes transformation by ras. Cell. 76:747-760.

33. Cavigelli, M., F. Dolfi, F.X. Claret, and M. Karin. 1995. Induction of c-fos expression through JNK-mediated TCF/Elk-1 phosphorylation. EMBO
(Eur. Mol. Biol. Organ.) J. 14:5957-5964.

34. Tremble, P., C.H. Damsky, and Z. Werb. 1995. Components of the nuclear signaling cascade that regulate collagenase gene expression in response to integrin-derived signals. J. Cell Biol. 129:1707-1720.

35. Deng, T., and M. Karin. 1993. JunB differs from c-Jun in its DNA-binding and dimerization domains, and represses c-Jun by formation of inactive heterodimers. Genes Dev. 7:479-490.

36. Lohnes, D., M. Mark, C. Mendelsohn, P. Dolle, D. Decimo, M. LeMeur, A. Dierich, P. Gorry, and P. Chambon. 1995. Developmental roles of the retinoic acid receptors. J. Steroid Biochem. Mol. Biol. 53:475-486.

37. Chen, J.Y., S. Penco, J. Ostrowski, P. Balaguer, M. Pons, J.E. Starrett, P. Reczek, P. Chambon, and H. Gronemeyer. 1995. RAR-specific agonist/antagonists which dissociate transactivation and AP1 transrepression inhibit anchorage-independent cell proliferation. EMBO (Eur. Mol. Biol. Organ.) J. 14:11871197.

38. Pfahl, M. 1993. Nuclear receptor/AP-1 interaction. Endocr. Rev. 14: 651-658.

39. Schule, R., P. Rangarajan, N. Yang, S. Kliewer, L.J. Ransone, J. Bolado, I.M. Verma, and R.M. Evans. 1991. Retinoic acid is a negative regulator of AP1-responsive genes. Proc. Natl. Acad. Sci. USA. 88:6092-6096.

40. Treier, M., L.M. Staszewski, and D. Bohmann. 1994. Ubiquitin-dependent c-Jun degradation in vivo is mediated by the $\delta$ domain. Cell. 78:787-798.

41. Musti, A.M., M. Treier, and D. Bohmann. 1997. Reduced ubiquitindependent degradation of c-Jun after phosphorylation by MAP kinases. Science. 275:400-402.

42. Tavakkol, A., C.C. Zouboulis, E.A. Duell, and J.J. Voorhees. 1994. A retinoic acid-inducible skin-specific gene (RIS-1/psoriasin): molecular cloning and analysis of gene expression in human skin in vivo and cultured skin cells in vitro. Mol. Biol. Reports. 20:75-83. 